\title{
Medidas de rede e apoio social no Estudo Pró-Saúde: pré-testes e estudo piloto
}

\author{
Social network and social support measures from \\ the Pró-Saúde Study: pre-tests and pilot study
}

Dóra Chor 1

Rosane Harter Griep 2

Claudia S. Lopes 3

Eduardo Faerstein 3

\footnotetext{
1 Departamento de Epidemiol ogi a e Métodos Quantitativos em Saúde, Escola Nacional deSaúde Pública, Fundação Oswaldo Cruz. Rua Leopoldo Bulhões 1480, Rio de Janeiro, RJ 21041-210, Brasil.

2 Departamento de Enfermagem em Saúde Pública, Escola de Enfermagem Anna Nery, Universidade Federal do Rio de Janeiro. Rua Afonso Cavalcante 275, Rio de Janeiro, RJ 20211-110, Brasil.

3 Departamento de Epidemiologia, Insti tuto de Medicina Social, Universi dade do Estado do Rio de Janeiro. Rua São Francisco Xavier 524 7o andar, Rio de Janeiro, RJ 20559-900, Brasil.
}

Abstract We describe methodol ogical steps in the sel ection of questions on social networksand support for a cohort study of 4,030 employees from a public university in Rio de Janeiro. First, group discussions with volunteers were conducted to explore the adequacy of related concepts. Next, questions in the Medical Outcomes Study question naire were submi tted to standard "forward-" and "back-translation" procedures. The questions were subsequently eval uated through fivestages of pre-tests and a pil ot study. No question had a proportion of non-response greater than $5 \%$. Pearson correlati on coefficients between questions were distant from both zero and unity; correlation between all items and their dimension score was higher than 0.80 in most cases. Finally, Cronbach Alpha coefficients were above 0.70 wi thin each dimension. Results suggest that social networks and support will be adequately measured and will allow for the investigation of their associations with health outcomes in a Brazilian population.

Key words Social Support; Questionnaires; Cohort Studies

Resumo Nesteartigo, relatamos a metodologia de sel eção de perguntas sobre rede e apoi o social, incluídas em um estudo de coorte de 4.030 funcionários de uma universidade pública no Rio de Janeiro. Em primei ro lugar, a adequação de concei tos foi explorada em discussões de grupos de voluntários. Em segui da, o questionário do Medical Outcomes Study foi submetido a procedimentos padronizados de tradução e versão. As pergu ntas foram a seguir avali adas em cinco etapas de pré-testes e estudo piloto. Nenhuma pergunta apresentou proporção de não-resposta acima de $5 \%$. Os coefici entes de correl ação de Pearson entre os itens foram distantes de zero e da unidade; a correl ação entre cada item e o escore de sua dimensão foi superior a 0,80 em quase todos os casos. Final mente, os coeficientes Al pha de Cronbach foram superi ores a 0,70 em todas as dimensões. Os resul tados sugerem que aspectos de rede e a poio social serão mensu rados adequadamente, permitindo a investi gação de suas associações com desenlaces relacionados à saúdeem um grupo populacional no Brasil.

Palavras-chave Apoio Social; Questionário; Estudos de Coortes 
Introdução

Nas últimas décadas, estados subjetivos como depressão e solidão, atitudes rel acionadas à sexualidade e à qualidade de vida passaram a ser intensivamente investigados em estudos epidemiológicos. Foi necessário então utilizar metodologias, já desenvolvidas principalmente nas áreas da educação e da psicologia, para mensurar conceitos que não são passíveis de tradução em valores observáveis por meio da posição de um ponteiro (Streiner \& Norman, 1989). Dentre esses conceitos destaca-se o de laços sociais.

A relação entre laços sociais e saúde foi sugerida, na década de 70, por Sidney Cobb (1976) e, de maneira independente, por John Cassel (1976), quando compilaram evidências de que a ruptura de laços sociais aumentava a suscetibilidade a doenças. Diversos pesquisadores notaram que portadores de enfermidades tão diversas como hipertensão arterial, depressão e tuberculose, e ainda vítimas de acidentes, relatavam com maior freqüência o fato de não estarem (ou não se sentirem) inseridos em uma rede de apoio mútuo, ou ainda de terem experimentado em maior grau perdas importantes de laços sociais (e.g. viuvez, separação amorosa, desemprego, mudança de moradia) (Pilisuk $\&$ Minkler, 1985)

Dentre as diferentes idéias englobadas na expressão "laços sociais", dois conceitos se destacam: rede social e apoio social. As redes (networks) são definidas como "teias" de relações sociais que circundam o indivíduo bem como suas características (por exemplo, disponibilidade e freqüência de contato com amigos e parentes), ou como os grupos de pessoas com quem há contato ou al guma forma de participação social (por exemplo, grupos religiosos, associações sindicais) (Berkman \& Syme, 1979; Bowling, 1997). Além desses dois aspectos, Hanson \& Östergren (1987), adi cionaram a “ancoragem social" (social anchorage), que descreve a percepção do grau de identificação ou vínculo entre o indivíduo e os grupos formais e informais a que pertence (ambiente de trabalho, área de moradia, família, por exemplo).

A definição de apoio social não é consensual. Segundo Bowling (1997), Sherbourne \& Stewart (1991) e Cohen \& Wills (1985), trata-se do grau com que relações interpessoais correspondem a determinadas funções (por exemplo, apoio emocional, material e afetivo), com ênfase no grau de satisfação do indivíduo com a disponibilidade e qualidade dessas funções. Cobb (1976) define apoio social como a informação que leva o indivíduo a acreditar que é querido, amado e estimado, e que faz parte de uma rede social com compromissos mútuos. Minkler (1985) ressalta que apoio social deve ser compreendido como um processo recíproco, isto é, que gera efeitos positivos tanto para quem recebe como para quem oferece o apoio, permitindo que ambos tenham maior sensação de controle sobre suas vidas.

Embora os mecanismos de ação exercidos pela rede e apoio social nos sistemas de defesa do organismo humano ain da não tenham sido elucidados, duas hipóteses básicas são apresentadas. Na primeira, atuariam "tamponan do" a resposta do organismo em forma de doença, que pode ocorrer em conseqüência de grandes perdas ou rupturas emocionais (Cohen $\&$ Wills, 1985). Na segunda hipótese, o apoi o social poderia reforçar a sensação de controle sobre a própria vida, o que por sua vez implicaria em efeitos positivos sobre a saúde (Rodin, 1986).

A forte e consistente associação inversa entre um índice multidimensional de laços sociais e a taxa geral de mortalidade, foi um dos primei ros efeitos identificados da rede e apoio social sobre a saúde (Berkman \& Syme 1979). Investigações posteriores confirmaram a relação inversa entre a magnitude de índices de rede e apoio social e o risco de morrer por doença coronariana, acidente vascular cerebral e também por neoplasias malignas (Dressler et al., 1997; Dalgard \& Haheim, 1998).

Além da forte relação com a redução da mortalidade, dimensões de rede e de apoio social associam-se também, de forma direta, com a sobrevida após diagnósticos de doença coronariana, câncer e acidente vascular cerebral (Vogt et al., 1992), e de maneira inversa com a incidência de insônia (Hanson \& Ostergren, 1987), com a freqüência de hipertensão arterial (Strogatz \& James, 1986), com a aquisição dehábitos como o tabagismo e consumo de álcool (Westman et al., 1985), e também com o risco de demência (Fratiglione et al., 2000).

Investigações a respeito de rede e apoio social dizem respeito a perguntas extremamente relevantes para o desenvolvimento de políticas e programas de saúde, apesar das inconsistências conceituais ainda existentes e das dificuldades de transformação desses conceitos em variáveis (Dean et al., 1994; O'Reilly, 1988). Tais dificuldades podem explicar parcialmente a tendência a priorizar a pesquisa de fatores de risco biológico e hábitos relacionados à saúde, na pesquisa etiológica, ao invés do ambiente psicossocial. No entanto, não há dúvida de que a saúde - individual ou coletiva - resulta de relações complexas entre inúmeros fatores biológicos, psicológicos e sociais. Para compreen- 
der essas relações, problemas metodológicos existentes devem ser enfrentados.

Neste artigo, relatamos as etapas iniciais percorridas pelos pesquisadores do Estudo Pró-Saúde, entre setembro de 1998 e junho de 1999, para incluir questões relativas à rede e apoio social em um questionário multidimensional acerca de aspectos da saúdefísica e mental dos funcionários técnico-administrativos de uma universidade pública, no Rio de Janeiro (Figura 1).

Encontra-se em preparação outra comunicação, na qual relatamos os resultados dos estudos de confiabilidade e validade do instrumento.

\section{Metodologia}

População e delineamento do estudo

O Pró-Saúde constitui um estudo de coorte de 4.030 funcionários técnico-administrativos do quadro efetivo da Universidade, que responderam a um questionário auto-preenchido na Fase 1 de coleta de dados, en tre agosto e novembro de 1999, e cujos resultados formarão a “linha de base" da investigação. O seguimento da coorte incluirá contatos bienais com os participantes, voltados para a avaliação de desfechos em estudo, além da complementação e atual ização de dados de base.

\section{Pesquisa bibliográfica}

A multiplicidade de índices que pretendem medir estados subjetivos, como rede e apoio social, é um fato observado com freqüência. Mesmo assim, havia a possibilidade de que a validade de face e de conteúdo desses instrumentos não fossem consideradas adequadas à população desse estudo, situação que demandaria a elaboração de um novo índice. Streiner \& Norman (1989) comentam que a construção de um índice válido e confiável não é uma tarefa trivial, sendo recomendada a utilização de índices previamente elaborados e testados, sempre que possível.

Em uma primeira etapa, realizamos pesquisa bibliográfica, onde foi possível identificar apenas um instrumento brasileiro para avaliação quantitativa de rede e apoio social, cujo conteúdo era especificamente dirigido ao período de gestação, parto e puerpério (Langer et al., 1996; Victora et al., 1994). Nesta revisão foram identificados diferentes conceitos (vide introdução) e instrumentos de medida de rede e apoio social, utilizados por pesquisadores de diversos países, cujas abordagens variam bas- tante tanto no conteúdo quanto na forma das perguntas aplicadas (McDowell \& Newell, 1996). Decidiu-se pela necessidade de explorar, entre brasileiros, a pertinência de conceitos, funções e componentes de rede e de apoio social antes de optar e/ou adaptar um dos questionários identificados. Para isso, aplicou-se técnica qualitativa de discussão em grupo, na qual utilizamos alguns procedimentos adotados em grupos focais e outros adotados em grupos naturais, de acordo com Coreil (1995).

\section{Discussão em grupo}

Duas sessões de discussão em grupo com duração de duas horas e meia cada uma, foram realizadas com funcionários técnico-administrativos de diversas unidades da Fundação Oswal do Cruz (FIOCRUZ), com perfil semelhante ao da população de estudo. Os grupos foram constituídos, respectivamente, por doze muIheres e doze homens, observando-se adicionalmente critérios de homogeneidade entre os participantes quanto a idade e nível sócio-econômico. A exposição de opiniões divergentes foi estimulada, sem necessidade de atingir um consenso a respeito do assunto.

De maneira resumida, os seguintes tópicos fizeram parte do roteiro de discussão:

a) Escreva as iniciais e o tipo de relacionamento das pessoas que são importantes na sua vida;

b) Escreva as iniciais e o tipo de relacionamento de pessoas que você considera que são íntimas;

c) O que você acha que diferenciou as duas listas de pessoas, as importantes e as íntimas?

d) Que tipo de ajuda você já precisou das pessoas com quem você se relaciona?

e) Por favor, lembre de situações em que você já precisou de ajuda material de pessoas que não moram com você. Por exemplo, se você já precisou de $\mathrm{R} \$ 10,00$ emprestados ou se já precisou deixar seu filho com alguém durante algumas horas (situações semelhantes foram apresentadas em relação a apoio emocional e deinformação).

f) Quais são os grupos de pessoas com quem você se reúne normalmente (por exemplo, na igreja, em grupos de oração, no futebol)? Que importância esses grupos têm na sua vida?

Após a discussão de cada grupo, onde participaram um coordenador e um redator, as anotações realizadas por este, bem como a transcrição das fitas gravadas foram analisadas, de forma independente, por dois dos autores (DC e RHG). Desejava-se explorar, entre outros aspectos, abordagens diferentes, utili- 
Questionário

Perguntas do bloco de rede social

D1 - Com quantos parentes você se sente à vontade e pode falar sobre quase tudo?

(Se for o caso, inclua esposo(a), companheiro(a) ou filhos nesta resposta.) parentes; $\square$ nenhum

D2 - Com quantos amigos você se sente à vontade e pode falar sobre quase tudo?

(Não inclua esposo (a), companheiro(a) ou filhos nesta resposta.) amigos; $\square$ nenhum

D3 - Nos últimos 12 meses, você participou de atividades esportivas em grupo (futebol, vôlei, basquete, outros) ou atividades artísticas em grupo (grupo musical, coral, artes plásticas, outras)?

D4 - Nos últimos 12 meses, você participou de reuniões de associações de moradores ou funcionários, sindicatos ou partidos?

D5 - Nos últimos 12 meses, você participou de trabalho voluntário não remunerado, em organizaçõ es não governamentais (ONGs), de caridade, ou outras?

As seguintes opções de resposta foram oferecidas para as perguntas D3, D4 e D5

sim;

$$
\text { não }
$$

Se SIM, com que freqüência?

mais de uma vez por semana

uma vez por semana

2 a 3 vezes por semana

algumas vezes no ano

uma vez no ano

Perguntas do bloco de apoio social

Todas as perguntas foram precedidas da expressão: Se você precisar...com que freqüência conta com alguém

D6 - que o ajude, se ficar de cama?

D7 - para Ihe ouvir, quando você precisa falar?

D8 - para Ihe dar bons conselhos em uma situação de crise?

D9 - para levá-lo ao médico?

D10 - que demonstre amor e afeto por você?

D11 - para se divertir junto?

D12 - para lhe dar informação que o(a) ajude a compreender uma determinada situação?

D13 - em quem confiar ou para falar de você ou sobre seus problemas?

D14 - que the dê um abraço?

D15 - com quem relaxar?

D16 - para preparar suas refeições, se você não puder prepará-las?

D17 - de quem você realmente quer conselhos?

D18 - com quem distrair a cabeça?

D19 - para ajudá-lo nas tarefas diárias, se você ficar doente?

D20 - para compartilhar suas preocupações e medos mais íntimos?

D21 - para dar sugestões sobre como lidar com um problema pessoal?

D22 - com quem fazer coisas agradáveis?

D23 - que compreenda seus problemas?

D24 - que você ame e que faça você se sentir querido?

As seguintes opções de resposta foram oferecidas para todas as perguntas:

nunca; $\square$ raramente; $\square$ às vezes; $\square$ quase sempre; $\square$ sempre 
zadas por instrumentos diversos, como por exemplo perguntas relacionadas ao número e freqüência de contato com pessoas íntimas (closefriends/relatives) (Berkman \& Syme, 1979; Östergren et al., 1991), em contraposição a pessoas importantes (significant people) (Norbeck \& Tilden, 1983; Penninx et al., 1997). Foi possível detectar os distintos significados desses conceitos para os participantes. Na lista de pessoas importantes foram incluídos não só parentes, amigos, colegas de trabalho, comadres, compadres, afilhados como também: “...o pessoal do salão onde eu faço a unha, eu adoro eles...vai todo mundo no mesmo horário...aquela coi sa gostosa".

Outro aspecto interessante foi a inclusão por alguns homens e algumas mulheres - entre as pessoas importantes mas não entre as íntimas - de parentes já falecidos, do santo protetor e mesmo de Deus: “....mi nha avó já falecida...eu tinha uma afinidade mui to grandecom el a...então eu a sinto presente, perto de mim... não é conversar diretamente, mas parece...", “...marido, mãe,eu, eu mesma, o meu interior, o meu Deus interior".

De acordo com os depoimentos, foi possível perceber que as pessoas consideradas importantes eram aquelas percebidas como estando presentes na vida dos participantes (fisicamente ou não) ou que compartilhavam momentos de al egria, entre outros aspectos.

A lista de pessoas íntimas incluiu tipos semelhantes de relacionamento - parentes e amigos - mas foi, em todos os casos, muito menor do que a lista de pessoas importantes: “....nas pessoas importantes eu tinha listado doze pessoas,enxuguei, e dessas doze, as pessoas ínti mas são três, né? E eu acho que pessoa íntima tem uma relação assim...ajuda a solucionar proble mas meus,e eu também ajudo a essas pessoas todas a resolver os problemas delas.E a intimidade está um pouco ligada a isso...solução de problemas, atéfunciona assi m como confidente, né?"

Uma das características que distinguiram o grupo dos "íntimos", foi a confiança para contar tudo: “...divido tudo, todos os meus problemas, e el es sempre me socorrem...; .....tenho irmãos que eu gosto muito, que são importantes prá mim...todos são da minha família,mas não étudo que a gente pode contar prá eles, entendeu?"

Não somente o sentimento de confiança mas também o de sentir-se à vontade para falar abertamente sobre todo e qualquer assunto fizeram parte do conceito de intimidade para vários participantes: “....a questão da intimidadenão é simplesmentevocêconfiar ou não confiar na pessoa, né? Masé porqueeu mesinto maisà vontade para falar, para conversar mais abertamente...".
Corroborando essa distinção, al guns participantes excluíram seus cônjuges da lista de pessoas íntimas: “...nem tudo se pode falar pro marido", “...eu tire da intimidadeaqui, a minha esposa, (estava incluída na lista das pessoas importantes) porquea genteé recém-casado....acho quea única pessoa que sabe tudo praticamente de mim é um amigo meu...porque tem coisas queeu prefiro queuma pessoa não saiba...".

A reciprocidade de confiança e para resolução de problemas (presente em depoimentos anteriores), aparece também na profundidade de conhecimento entre as pessoas íntimas: “...eu conheço ele tanto quanto el e me conhece, por essa intimidade..."; “....pessoa íntima é aquela que meconhece, às vezes, mais do que eu mesma....éaquelequeeu não posso nem fugir, é o íntimo mesmo".

Além das diferenças entre pessoas íntimas e importantes, os tipos de ajuda ou apoio - material, emocional, entre outros - que fazem parte das medidas de apoio social utilizadas em instrumentos identificados na literatura internacional, foram também aval iados com o intuito de explorar sua adequação em população com características semel hantes àquela do Estudo Pró-Saúde. Um dos participantes, declarou precisar de “...apoio moral, espiritual efinancei ro...". Outros detal haram esses tipos gerais, predominando necessidades e expectativas relacionadas ao "apoio moral", além do financeiro. Dessa forma, citaram necessitar de: “...um conselho, uma opinião...";“...honesti dade,fidelidade, uma palavra de apoi o..."; “...quea pessoa seja um bom ouvinte, aconchego ecarinho".

A análise das discussões em grupo teve efeito direto na formulação das perguntas. Quanto à rede social, considerou-se mais importante obter informação sobre a quantidade de amigos e parentes íntimos, de acordo com Östergren et al. (1991) e Berkman \& Syme (1979) do que a informação sobre pessoas importantes. Com essa finalidade, utilizamos conceitos adotados por esses autores e confirmados pelas discussões em grupo para a formulação das perguntas (Com quantos parentes/amigos você se sente à vontade e pode falar sobre quase tudo? Vide texto integral a seguir). Além disso, decidiu-se enfatizar as medidas de apoio social (vide tópico Pré-testes), assim como foi ratificada a relevância dos tipos de apoio avaliados em outros instrumentos, incluindo-se itens relativos a apoio material, emocional, de informação, afetivo e de interação positiva.

Ao finalizarmos a análise das discussões em grupo, adotamos o conceito de rede social proposto por Berkman \& Syme (1979) e Bowling (1997), segundo o qual trata-se de relações so- 
ciais que circundam o indivíduo e os grupos de pessoas com quem há contato ou alguma forma de participação social. Apoio social foi definido como o grau com que relações interpessoais correspondem a determinadas funções (por exemplo, apoio emocional, material e afetivo), com ênfase no grau de satisfação do indivíduo com a disponibilidade e qualidade dessas funções segundo Cohen \& Wills (1985), Sherbourne \& Stewart (1991) e Bowling (1997). De acordo com os conceitos adotados, propusemos a versão do questionário a ser aplicada nos pré-testes.

\section{Pré-testes}

O objetivo principal dos pré-testes foi o aprimoramento das sucessi vas versões do questionário. O relato detalhado da estratégia utilizada nos pré-testes encontra-se em outra publicação (Faerstein et al., 1999). Resumidamente, foram desenvolvidos em cinco estágios, que envolveram cerca de 150 voluntários.

$\mathrm{Na}$ etapa 1, a própria equipe de pesquisadores e colaboradores respondeu à primeira versão, subsidiando com comentários e sugestões, a elaboração da segunda versão do questionário. Nas etapas 2 a 5 , os pré-testes foram realizados com voluntários, funcionários técnico-administrativos da FIOCRUZ, Universidade Federal do Rio de Janeiro (UFRJ) e Secretaria de Saúde e Higiene do Estado do Rio de Janeiro.

Diferentes estratégias foram aplicadas nas etapas de pré-testes. $\mathrm{Na}$ etapa 2 , os respondentes foram entrevistados e estimulados a pensar al to e a tecer comentários detalhados sobre qual quer tipo de dificuldade encontrada. $\mathrm{Na}$ etapa 3, a terceira versão do questionário foi, pela primeira vez "aplicada", ou seja, todo o questionário foi preenchido por 25 voluntários; nessa etapa os aplicadores limitaram-se a registrar dúvi das e questionamentos que surgiram espontaneamente. Nas duas últimas etapas, a diagramação definitiva do questionário foi gradativamente delineada, assim como o contexto de aplicação simultânea em grupos de participantes.

A análise dos relatórios das três primeiras etapas dos pré-testes revelou, entre outros aspectos, a necessidade de redução do tempo médio de preenchimento. Assim, todo o questionário foi revisto, buscando-se simplificar e até excluir itens. No bloco relativo à rede e apoio social, onde as perguntas utilizadas até essa etapa, timham sido adaptadas do questionário proposto por Hanson \& Östergren (1987), as questões referentes à rede eram mais nume- rosas do que aquelas de apoio social. Em função da necessidade de encurtar todo o questionário e, especialmente, em função da análise das discussões em grupo (vide item Discussões em Grupo), que revelou a maior importância atribuída à disponibilidade e qualidade das funções de apoio social, optou-se pela aplicação do conjunto dos itens utilizados no Medical Outcomes Stu dy (MOS) (Sherbourne \& Stewart, 1991). Nesse instrumento, os itens relativos a apoio social são priorizados, em comparação com os de rede, agrupando-se suas diferentes funções em cinco dimensões: emocional, de informação, material, afetiva e de interação positiva (vide texto integral a seguir).

Os itens utilizados no MOS são também aplicados, atualmente, no National Population Health Study (NPHS), estudo anual sobre as con dições de saúde da população canadense. A experiência dos pesquisadores responsáveis pelo NPHS também reforçou nossa escolha já que, segundo depoimento colhido, após diversas tentativas com diferentes instrumentos de medida de apoio social, o questionário utilizado pelo MOS foi o que possibilitou a melhor discriminação de subgrupos da população canadense em relação a desfechos na área da saúde (Gary Catlin, comunicação pessoal, 1999).

No questionário utilizado no MOS, cada uma das cinco dimensões de apoio social é constituída por diversas perguntas, perfazendo um total de dezenove. As perguntas são introduzidas pela frase "se você precisar..." seguida pelo tipo de apoio, e as opções de resposta são apresentadas da mesma forma para todos os itens: nunca, raramente, às vezes, quase sempre, sempre (vide texto integral a seguir).

A validade de face e a validade de conteúdo dos itens de apoio social do MOS foram consideradas adequadas pelos investigadores do Estudo Pró-Saúde. Ambos os tipos de validade representam julgamentos subjetivos a respeito da capacidade do índice de avaliar as qualidades do conceito que se deseja medir (daí a denominação "de face" ou seja, se o instrumento "parece" medir aquilo a que se propõe) e, no caso da validade de conteúdo, quanto à possibilidade de se abranger, no instrumento, todos os aspectos ou dimensões considerados relevantes ao conceito em estudo (Streiner \& Norman, 1989). Cabe notar que esse julgamento só foi possível em função da realização prévia das discussões em grupo, onde pudemos nos aproximar da relevância e do significado de alguns conteúdos, englobados nos conceitos de rede e apoio social, para um conjunto de pessoas com características semelhantes às da populaçãoalvo. 
Quanto à validade de constructo desse instrumento, Sherbourne \& Stewart (1991) identificaram alta correlação entre as dimensões de apoio social e outros conceitos que, teoricamente, estão relacionados. Assim, altos coeficientes de correlação foram estimados entre as dimensões de apoio social e medidas de solidão (correlação negativa); com avaliações de dinâmica familiar e conjugal, e também com medidas de saúde mental (correlação positiva). Além disso, os bons resultados observados pelos autores em relação à consistên cia interna (coeficientes Alpha de Cronbach acima de 0,91) e à estabilidade das medidas após um ano (coeficientes de estabilidade acima de 0,72 ), para as dimensões de apoio social, também reforçaram a escolha do questionário aplicado no MOS, submetido, em seguida, ao processo de tradução e versão.

Tradução e versão do instrumento: buscando a equivalência

Buscando al cançar a maior equivalência possível entre instrumentos aplicados em idiomas diferentes (o original em inglês e sua tradução para o português), um processo de tradução e versão (forward and backtranslation) (Herdman et al., 1997) foi realizado. Este consistiu na tradução do original em inglês para o português, por um tradutor profissional, e da versão, outra vez para o inglês, por outro tradutor igualmente qualificado. A segunda versão em ingl ês foi comparada à original por cinco epidemiologistas fluentes nos dois idiomas, que consideraram equivalentes as duas versões em inglês.

Todas as perguntas utilizadas no MOS, traduzidas para o português e sem nenhuma adaptação, foram inseridas no questionário global e submetidas às duas últimas etapas dos pré-testes e também ao estudo piloto.

Estud o piloto

O estudo piloto representou um "ensaio geral" de todas as atividades previstas para a coleta de dados propriamente dita. Uma amostra sistemática de 200 funcionários administrativos em um total de cerca de 2.000 - contratados em regime de prestação de serviços fizeram parte dessa etapa, por apresentarem um perfil social e funcional semelhante ao da população de estudo, ou seja, dos funcionários efetivos da Universidade.

A análise dos resultados do estudo piloto identificou proporção muito pequena de dados ausentes no bloco relativo à rede e apoio social: em duas perguntas, houve oito (4\%) e dez (5\%) respostas ausentes, respectivamente; em uma pergunta, houve duas ( $1 \%$ ) respostas ausentes; e em cinco perguntas, houve uma $(0,5 \%)$ resposta ausente. Assim, dentre os vinte e quatro itens do bloco, dezesseis itens (66\%) não apresentaram nenhum dado ausente.

Al ém da verificação da quanti dade de dados ausentes, o que poderia sugerir dificuldades de compreensão da pergunta, aplicamos outros critérios de avaliação da qualidade dos itens que fizeram parte do bloco de apoio social.

Em primeiro lugar, verificamos o grau de correlação entre cada uma das perguntas e todas as outras, através do coeficiente de correlação de Pearson, que é um indicador da validade de discriminação ou seja, avalia a capacidade dos itens em medir apoio social e não qualquer outro conceito (Tabela 1). Nenhum dos itens apresentou coeficiente de correlação de Pearson próximo de zero ou próximo da unidade com muitos outros itens, o que favoreceria sua exclusão por ausência de relação com o conceito medido (no primeiro caso), ou por redundância com outros itens (no segundo caso) (Rowland et al., 1991).

A segunda etapa da avaliação do bloco de apoio social foi realizada através do coeficiente de correlação de Pearson entre cada pergunta e a dimensão a que teoricamente pertence. Para compor a medida de cada dimensão de apoio social (apoio afetivo, emocional, informação, interação positiva e material), cinco escores foram elaborados. Assim, tomando como exemplo as três perguntas que compõem a dimensão apoio afetivo (Se você precisar...com que freqüência (1) conta com al guém que demonstre amor e afeto por você; (2) conta com alguém que lhe dê um abraço; (3) conta com alguém que você ame e que faça você se sentir querido), suas respostas foram codificadas entre 1 ("nunca") e 5 ("sempre"), com pontuações intermediárias para "raramente", "às vezes" e “quase sempre". Efetuou-se a soma dos "pontos obtidos" nessas três perguntas. Por exemplo, se um funcionário respondeu "quase sempre" em dois itens, e "sempre" no terceiro, seu total de pontos foi igual a treze. O resultado dessa adição foi dividido pelo número máximo de pontos que o respondente poderia totalizar segun do o número de perguntas efetivamente respondidas (não deixadas em branco). Assim, aqueles que responderam às três perguntas da dimensão apoio afetivo, poderiam perfazer, no máximo, quinze pontos $(5 \times 3)$. O resultado da razão - total de pontos obtidos/máximo de pontos possíveis - foi multiplicado por 100, a fim de padronizar a variação dos escores das cinco dimensões entre zero e 100, independen- 
Tabela 1

Coeficientes de correlação de Pearson entre as perguntas do bloco de apoio social. Estudo Pró-Saúde - Piloto, 1999.

\begin{tabular}{|c|c|c|c|c|c|c|c|c|c|c|c|c|c|c|c|c|c|c|c|}
\hline & D6 & D7 & D8 & D9 & D10 & D11 & D12 & D13 & D14 & D15 & D16 & D17 & D18 & D19 & D20 & D21 & D22 & D23 & D24 \\
\hline D6 & 1,00 & & & & & & & & & & & & & & & & & & \\
\hline D7 & 0,639 & 1,00 & & & & & & & & & & & & & & & & & \\
\hline D8 & 0,478 & 0,794 & 1,00 & & & & & & & & & & & & & & & & \\
\hline D9 & 0,601 & 0,538 & 0,590 & 1,00 & & & & & & & & & & & & & & & \\
\hline D10 & 0,505 & 0,533 & 0,513 & 0,539 & 1,00 & & & & & & & & & & & & & & \\
\hline D11 & 0,490 & 0,523 & 0,444 & 0,462 & 0,528 & 1,00 & & & & & & & & & & & & & \\
\hline D12 & 0,505 & 0,670 & 0,650 & 0,537 & 0,621 & 0,647 & 1,00 & & & & & & & & & & & & \\
\hline D13 & 0,527 & 0,759 & 0,665 & 0,525 & 0,569 & 0,558 & 0,707 & 1,00 & & & & & & & & & & & \\
\hline D14 & 0,489 & 0,620 & 0,544 & 0,512 & 0,647 & 0,599 & 0,654 & 0,619 & 1,00 & & & & & & & & & & \\
\hline D15 & 0,490 & 0,554 & 0,498 & 0,450 & 0,606 & 0,781 & 0,709 & 0,623 & 0,689 & 1,00 & & & & & & & & & \\
\hline D16 & 0,177 & 0,347 & 0,349 & 0,303 & 0,310 & 0,408 & 0,344 & 0,258 & 0,396 & 0,415 & 1,00 & & & & & & & & \\
\hline D17 & 0,456 & 0,630 & 0,625 & 0,487 & 0,541 & 0,537 & 0,733 & 0,742 & 0,584 & 0,629 & 0,397 & 1,00 & & & & & & & \\
\hline D18 & 0,372 & 0,500 & 0,530 & 0,371 & 0,518 & 0,759 & 0,652 & 0,584 & 0,546 & 0,756 & 0,471 & 0,722 & 1,00 & & & & & & \\
\hline D19 & 0,480 & 0,614 & 0,548 & 0,538 & 0,473 & 0,440 & 0,582 & 0,573 & 0,606 & 0,497 & 0,558 & 0,658 & 0,496 & 1,00 & & & & & \\
\hline D20 & 0,492 & 0,644 & 0,572 & 0,472 & 0,549 & 0,527 & 0,674 & 0,701 & 0,600 & 0,601 & 0,344 & 0,647 & 0,616 & 0,608 & 1,00 & & & & \\
\hline D21 & 0,454 & 0,691 & 0,707 & 0,510 & 0,491 & 0,479 & 0,658 & 0,712 & 0,549 & 0,597 & 0,253 & 0,693 & 0,580 & 0,552 & 0,745 & 1,00 & & & \\
\hline D22 & 0,435 & 0,558 & 0,534 & 0,405 & 0,548 & 0,747 & 0,658 & 0,604 & 0,641 & 0,813 & 0,505 & 0,691 & 0,829 & 0,555 & 0,658 & 0,619 & 1,00 & & \\
\hline D23 & 0,460 & 0,563 & 0,572 & 0,462 & 0,536 & 0,571 & 0,710 & 0,670 & 0,590 & 0,665 & 0,295 & 0,702 & 0,734 & 0,550 & 0,738 & 0,684 & 0,711 & 1,00 & \\
\hline D24 & 0,399 & 0,430 & 0,375 & 0,401 & 0,545 & 0,575 & 0,573 & 0,657 & 0,566 & 0,693 & 0,261 & 0,547 & 0,599 & 0,428 & 0,530 & 0,523 & 0,669 & 0,668 & 1,00 \\
\hline
\end{tabular}

Perguntas: Se você precisar, com que freqüência conta com alguém...

D6 - que o ajude, se ficar de cama?

D7 - para lhe ouvir, quando você precisar falar?

D8 - para lhe dar bons conselhos em uma situação de crise?

D9 - para levá-lo ao médico?

D 10 - que demonstre amor e afeto por você?

D 11 - para se divertir junto?

D12 - para lhe dar informação que o(a) ajude a compreender uma determinada situação?

D13 - em quem confiar ou para falar de você ou sobre seus problemas?

D14 - que lhe dê um abraço?

D15 - com quem relaxar?

D16 - para preparar suas refeições, se você não puder prepará-las?

D17 - de quem você realmente quer conselhos?

D18 - com quem distrair a cabeça?

D 19 - para ajudá-lo nas tarefas diárias, se você ficar doente?

D20 - para compartilhar suas preocupações e medos mais íntimos?

D21 - para dar sugestões sobre como lidar com um problema pessoal?

D22 - com quem fazer coisas agradáveis?

D23 - que compreenda seus problemas?

D24 - que você ame e que faça você se sentir querido?

temente do número de itens que compõem cada uma delas. Obteve-se assim os cinco escores, suas médias e desvios-padrão (Tabela 2).

Nenhuma das perguntas apresentou coeficiente de correlação menor do que 0,20 com o escore total de sua dimensão específica (Tabela 2), critério também preconizado para exclusão do item da respectiva dimensão (Rowland, 1991). Além disso, todos os itens de apoio afetivo, interação positiva e apoio material incluíram-se também no critério de composição de dimensões, proposto por Sherbourne \& Stewart (1991), segundo o qual o coeficiente de correlação do item com o escore de sua dimensão deve ser, pelo menos, dois desvios-padrão maior do que o mesmo coeficiente estimado com qualquer uma das outras dimensões (Tabela 2). Por outro lado, as perguntas que compõem a dimensão apoio emocional não se enquadraram neste último critério já que seus coeficientes de correlação com a dimensão apoio de informação foram também elevados. O mesmo resultado foi observado em relação às perguntas que compõem o apoio de informação, cujos coeficientes de correlação com o apoio emocional foram também elevados (Tabela 2). Procedimentos adicionais de análise serão utilizados a fim de explorar a possibilidade de que o apoio emocional e o apoio de informação sejam anal isados em conjunto, de acordo com o procedimento adotado por Sherbourne \& Stewart (1991), cujos resultados relaciona- 
Coeficientes de correlação de Pearson entre as perguntas do bloco de apoio social e respectivas dimensões. Estudo Pró-Saúde - Piloto, 1999.

\begin{tabular}{|c|c|c|c|c|c|c|c|}
\hline Disponibilidade de alguém para: & Média & Desvio-Padrão & $\begin{array}{l}\text { Apoio } \\
\text { Afetivo }\end{array}$ & $\begin{array}{l}\text { Apoio } \\
\text { Emocional }\end{array}$ & $\begin{array}{l}\text { Apoio de } \\
\text { Informação }\end{array}$ & $\begin{array}{l}\text { Interação } \\
\text { Positiva }\end{array}$ & $\begin{array}{l}\text { Apoio } \\
\text { Material }\end{array}$ \\
\hline Demonstrar amor e afeto por você - D10 & 4,61 & 0,81 & $0,844 *$ & 0,628 & 0,618 & 0,608 & 0,600 \\
\hline Dar-Ihe um abraço - D 14 & 4,48 & 0,90 & $0,868 *$ & 0,696 & 0,666 & 0,697 & 0,656 \\
\hline Amar - D24 & 4,44 & 0,94 & $0,838 *$ & 0,654 & 0,581 & 0,699 & 0,488 \\
\hline Ouvi-lo - D 7 & 4,24 & 1,12 & 0,613 & $0,850 *$ & 0,798 & 0,589 & 0,699 \\
\hline Confiar - D13 & 4,15 & 1,12 & 0,722 & 0,896* & 0,812 & 0,644 & 0,617 \\
\hline Dividir preocupações - D20 & 3,98 & 1,26 & 0,655 & $0,892 *$ & 0,761 & 0,643 & 0,628 \\
\hline Compreender seus probilemas - D23 & 4,07 & 1,07 & 0,706 & $0,849 *$ & 0,766 & 0,702 & 0,578 \\
\hline Dar-lhe bons conselhos - D8 & 4,22 & 1,08 & 0,552 & 0,745 & $0,853^{*}$ & 0,532 & 0,646 \\
\hline Dar-lhe informação - D12 & 4,16 & 1,02 & 0,723 & 0,791 & $0,864^{*}$ & 0,727 & 0,645 \\
\hline Dar-Ihe conselhos desejados - D17 & 4,01 & 1,15 & 0,655 & 0,779 & $0,877^{*}$ & 0,669 & 0,654 \\
\hline Dar sugestões - D21 & 4,01 & 1,26 & 0,608 & 0,814 & $0,889 *$ & 0,611 & 0,582 \\
\hline Divertir-se junto - D 11 & 4,27 & 1,05 & 0,671 & 0,623 & 0,600 & $0,912 *$ & 0,588 \\
\hline Relaxar - D 15 & 4,18 & 1,11 & 0,779 & 0,700 & 0,697 & 0,939* & 0,606 \\
\hline Fazer coisas agradáveis - D22 & 4,27 & 1,01 & 0,730 & 0,725 & 0,719 & $0,921 *$ & 0,620 \\
\hline Distrair a cabeça - D18 & 4,14 & 1,05 & 0,652 & 0,696 & 0,712 & $0,844^{*}$ & 0,558 \\
\hline Ajudá-lo se ficar de cama - D6 & 4,31 & 1,12 & 0,540 & 0,607 & 0,542 & 0,511 & $0,735^{*}$ \\
\hline Levá-lo ao médico - D9 & 4,07 & 1,28 & 0,561 & 0,573 & 0,608 & 0,476 & $0,811 *$ \\
\hline Preparar suas refeições - D 16 & 4,19 & 1,17 & 0,373 & 0,359 & 0,383 & 0,478 & $0,664^{*}$ \\
\hline Ajudar nas tarefas diárias - D19 & 4,13 & 1,18 & 0,585 & 0,674 & 0,672 & 0,537 & $0,842 *$ \\
\hline
\end{tabular}

* Itens que compõem cada dimensão.

dos a essas duas dimensões foram semelhantes aquel es encontrados em nosso estudo piloto.

Finalizando a etapa inicial de avaliação do questionário, os coeficientes Al pha de Cronbach foram estimados para avaliar se as diferentes perguntas utilizadas para avaliar o mesmo conceito - por exemplo, os três itens que supostamente medem o apoio afetivo - conseguem de fato alcançar esse objetivo (consistência interna) (Aday, 1991). Os coeficientes foram elevados, excedendo o padrão de 0,70 recomendado por Rowland (1991): 0,81, 0,89, 0,89, 0,93 e 0,76 respectivamente, para as dimensões de apoio afetivo, emocional, de informação, interação positiva e material.

\section{Comentários}

Apesar dos esforços realizados no Brasil nos últimos anos, no sentido de incorporar aspectos sócio-econômicos e sócio-culturais aos estudos epidemiológicos, dimensões relacionadas à rede e apoio social permanecem largamente inexploradas. De acordo com a revisão da literatura, a utilização de medidas de rede e/ ou apoio social, por meio de perguntas estrutura- das, parece estar limitada a en saio clínico com gestantes residentes em quatro municípios de países latinoamericanos (incluindo Pelotas, Rio Grande do Sul), onde os efeitos do reforço da rede social e do apoio emocional foram avaliados em relação à satisfação com a experiência da maternidade e aos cuidados com a saúde da mulher e da criança (Langer et al., 1996; Victora et al., 1994).

A construção de índices e escalas para medir fenômenos de natureza subjetiva não é uma tarefa fácil, recomendando-se a utilização de índices já existentes, desde que apresentem boa validade e confiabilidade. Essa foi a opção do grupo coordenador dessa pesquisa, embora o instrumento escolhido não tenha sido testado em população brasileira anteriormente. A validade de conteúdo dos itens que integram o questionário - que parecem se aplicar não só a populações norte-americanas mas também à população desse estudo, com bom nível de escolaridade - bem como os resultados dos prétestes e do estudo piloto, trouxeram maior segurança quanto a sua adequação à população de estudo. As estimativas de confiabilidade e validade do instrumento farão parte de sua avaliação em maior profundidade. 
Referências

ADAY, L. A., 1991. Designing and Conducting Health Surveys. A Compreehensive Guide. San Francisco: Jossey-Bass Publishers.

BERKMAN, L. F. \& SYME, S. L., 1979. Social networks, host resistance, and mortality: A nine-year follow-up study of Alameda County residents. American Journal of Epidemiology, 109:186-204.

BOWLING, A., 1997. Measuring social networks and social support. In: Measuring Health: A Review of Quality of Life Measurements Scales (A. Bowling, ed.), pp. 91-109, 2nd Ed., Buckingham: Open University Press.

CASSEL, J., 1976. The contribution of the social environment to host resistance. American Journal of Epidemiology, 104:300-314.

COBB, S., 1976. Social support as a moderator of life stress. Psychosomatic Medicine, 38:300-314.

COHEN, S. \& WILLS, T. A., 1985. Stress, social support and the buffering hypothesis. Psychol ogical Bulletin, 98:310-357.

COREIL, J., 1995. Group interview methods in community health research. Medical Anthropology, 16:193-210.

DALGARD, O. S. \& HAHEIM , L. L., 1998. Psychosocial risk factors and mortality: A prospective study with special focus on social support, social participation, and locus of control in Norway. Journal of Epidemiology and Community Health, 52: 476-481.

DEAN, K.; HOLST, E.; KREINER, S.; SCHOENBORN, C. \& WILSON, R., 1994. Measurement issues in research on social support and health. Journal of Epidemiology and Community Health, 48:201206

DRESSLER, W. W.; BALIEIRO, M. C. \& SANTOS, J. E., 1997. The cultural construction of social support in Brazil: Associations with health outcomes. Culture, Medicineand Psychiatry, 21:303-335.

FAERSTEIN, E.; LOPES, C. S.; VALENTE, K.; PLÁ, M. A. S. \& FERREIRA, M. B., 1999. Pré-testes de um questionário multidimensional autopreenchível: A experiência do Estudo Pró-Saúde. PHYSIS - Revista de SaúdeColetiva, 9:117-130.

FRATIGLIONE, L.; WANG, H.-X.; ERICSSON, K.; MAYTAN, M . \& WINBLAD, B., 2000. Influence of social network on occurrence of dementia: A community-based longitudinal study. Lancet, 355:13151319.

HANSON, B. S. \& OSTERGREN, P.-O., 1987. Different social network and social support characteristics, nervous problems and insomnia: Theoretical and methodological aspects on some results from population study "Men born in 1914", Malmo, Sweden. Social Science and Medicine, 25:849-859.

HERDMAN, M.; FOX-RUSHBY, J. \& BADIA, X., 1997. Equivalence and the translation and adptation of health-related quality of life questionnaires. Quality of Life Research, 6:237-247.

LANGER, A.; FARNOT, U.; GARCIA, C.; BARROS, F.; VICTORA, C.; BELIZAN, J. M. \& VILLAR, J., 1996. The Latin American trial of psychosocial support during pregnancy: Effects on mother's wellbeing and satisfaction. Social Sci ence and Medicine, 42: 1589-1597.
MCDOWELL, I. \& NEWELL, C., 1996. Measuring Health: A Guide to Rating Scales and Questionnaires. New York: Oxford University Press.

MINKLER, M., 1985. Building supportive ties and sense of community among the inner-city elderly: The Tenderloin Outreach Project. Health Educational Quarterly, 12:303-314.

NORBECK, J. S. \& TILDEN, V., 1983. Life stress, social support and emotional disequilibrium in complications of pregnancy: A prospective, multivariate study. Journal of Health Soci ety and Behavior, 24: 40-46.

O'REILLY, P., 1988. Methodological issues in social support and social network research. Social Scienceand Medicine, 26:863-873.

OSTERGREN, P.-O.; HANSON, B. S.; ISACSSON, S.-O. \& TEJLER, L., 1991. Social network, social support and acute chest complaints among young and middle-aged patients in an Emergency Department - A case-control study. Social Science and Medicine, 33:257-267.

PENNINX, B. W. J. H.; van TILBURG, T.; KRIEGSMAN, D. M. W.; DEEG, D. J. H.; BOEKE, A. J. P. \& van EIJK, J. T. M., 1997. Effects of social support and personal coping resources on mortality in older ages: The Longitudinal Aging Study Amsterdam. American Journal of Epidemiology, 146:510-519.

PILISUK, M. \& MINKLER, M., 1985. Supportive ties: A political economy perspective. Health Education Quarterly, 12:93-106.

RODIN, J., 1986. Aging and health: Effects of the sense of control. Science, 233:1271-1276.

ROWLAND, D.; ARKKELIN, D. \& CRISLER, L., 1991. Computer-Based Data Analysis. Using SPSS in the Social and Behavioral Sciences. Chicago: NelsonHall Inc.

SHERBOURNE, C. D. \& STEWART, A L., 1991. The MOS social support survey. Social Science and Medicine, 32:705-714.

STREINER, D. L. \& NORM AN, G. R., 1989. Health Measurement Scales. A Practical Guide to their Development and Use. Oxford: Oxford University Press.

STROGATZ, D. S. \& JAMES, S. A., 1986. Social support and hypertension among blacks and whites in a rural southern community. American Journal of Epidemiology, 124:949-956.

VICTORA, C.; LANGER, A.; BARROS, F.; BELIZAN, J .; FARNOT, U. \& VILLAR, J., 1994. The Latin American Multicenter Trial on psychological support during pregnancy: Methodology and baseline comparability. Controlled Clinical Trials, 15:379.

VOGT, T. M.; MULLOOLY, J. P.; ERNST, D.; POPE, C. R. $\&$ HOLLIS, J. F., 1992. Social Networks as predictors of ischemic heart disease, cancer, stroke and hypertension: Incidence, survival and mortality. Journal of Clinical Epidemiology, 45:659-666.

WESTMAN, M . \&EDEN, D., 1985. Shirom A. Job stress, cigarette smoking and cessation: The conditioning effects of peer support. Social Science and Medicine, 20:637-644. 